\title{
SIMULTANEOUS LOCALIZATION AND SEPARATION OF BIOMEDICAL SIGNALS BY TENSOR FACTORIZATION
}

\author{
Bahador Makki Abadi ${ }^{1,2}$, Delaram Jarchi ${ }^{1}$ and Saeid Sanei ${ }^{1}$ \\ ${ }^{1}$ Centre of Digital Signal Processing, School of Engineering, Cardiff University, Wales, UK \\ ${ }^{2}$ Electrical Engineering Department, Islamic Azad University, Ashtian Branch, Ashtian, IRAN
}

\begin{abstract}
In this paper, we introduce mathematical models based on multi-way data construction and analysis with a goal of simultaneously separating and localizing the sources in the brain by analysis of scalp electroencephalogram (EEG) data. we address the problem of EEG source separation and localization through a 3-way tensor analysis. We represent multi-channel EEG data using a third-order tensor with modes: space (channels), time samples and number of segments. Then we demonstrate that multi-way analysis techniques, in particular PARAFAC2, can successfully separate and localize disjoint sources within the brain. Also we used this method for separation of maternal and fetal ECG signals.
\end{abstract}

Index Terms - Blind Source Separation (BSS), Source Localization, PARAFAC, PARAFAC2, Tensor Factorization

\section{INTRODUCTION}

EEG [1] is a noninvasive measurement of electrical activity of the brain using electrodes placed on the scalp. The EEG reflects activation of the head musculature, eye movements, other physiological signals, interference from nearby electric devices, and changing conductivity in the electrodes due to the movements of the subject or physiochemical reactions at the electrode sites. These activities that are not directly related to the current cognitive processing of the subject are collectively referred to as background activities. Often in the presence of abnormalities such as tumor or epileptic seizure there are higher activities in some specific frequency bands and specific brain regions. These types of signals can also be detected and localized by processing the EEG signals.

Actually, separating and localizing normal EEGs can be used in many applications such as BCI (Brain Computer Interfacing) for detecting voluntary decisions and mental activities. On the other hand separating and localizing abnormal EEG signals is used in diagnosis of brain abnormalities and in junction with brain tomography[2][3].

In this paper we introduce a hybrid simultaneous source separation and 2-D localization method based on 3-way tensor factorization.

The remainder of the paper is structured as follows. In Section 2 the tensor factorization methods (PARAFAC-PARAFAC2) are described. In Section 3 EEG signals and their mixing concepts discussed and also PARAFAC2 method for simultaneous separation and localization is introduced. In Section 4 the results of applying the method to simulated and real data are provided. Finally Section 5 concludes the paper.

\section{PARAFAC AND PARAFAC2}

\subsection{An overview of three-way factorization methods}

Tensors represent datasets by preserving their multi-modal structures. Tensor decomposition methods, which are mostly based on generalizations of two-way factor models to higher-order datasets, can extract the true underlying structures of the data. Three-way data sets can be mainly classified as trilinear and non-trilinear. There are three-way factorizing methods for decomposition of trilinear data sets such as parallel factor analysis (PARAFAC) [4]. Recently, some algorithms such as PARAFAC2 [5][6] have been proposed to deal with non-trilinear data keeping uniqueness in solutions.

The fundamental expression of the PARAFAC model, which is used to describe decomposition of trilinear data sets, is given below:

$$
X_{i j k}=\sum_{r=1}^{R} A_{i r} F_{j r} C_{k r}+E_{i j k}
$$

where $X_{i j k}$ represents the $i, j, k$-th element in the three-way data set, $R$ is the number of components in common to the three modes, $A_{i r}, F_{j r}$ and $C_{k r}$ are respectively the elements in $A, F$ and $C$ used to obtain the $X_{i j k}$ elements, and $E_{i j k}$ is the residual term. Using matrix notation above equation can be presented as:

$$
X_{k}=F D_{k} A^{t}+E_{k}
$$

for $k=1, . . K$ where $(.)^{t}$ refers to transpose operation, $X_{k}$ represents the transposed $k$ th frontal slice of $X, A$ and $F$ are the component matrix in the first and second mode, respectively. $D_{k}$ is a diagonal matrix, whose diagonal elements correspond to the $k$ th row of the third component matrix $C$. Finally, $E_{k}$ contains the error terms corresponding to the entries in the $k$ th frontal slice.

PARAFAC2 is one of PARAFAC extensions and it supports variation in one mode of the tensor. In this paper we use PARAFAC2 as a powerful tool for signal processing specially for blind source separation and localization. Compared with PARAFAC, PARAFAC2 is designed to deal with non-trilinear data sets, while keeping uniqueness in the solutions, as the PARAFAC model does. To do so, PARAFAC2 allows a certain freedom in the shape of the $k$ slabs $\left(X_{k}\right)$ in the variable mode. To keep uniqueness in the solutions, all cross-product matrices $X_{k} X_{k}^{t}$ are forced to be constant over $k$, i.e. $X_{1} X_{1}^{t}=X_{2} X_{2}^{t}=X_{k} X_{k}^{t}$. Similar to PARAFAC equation (2), an alternative formulation of PARAFAC2 model in matrix notation is given as:

$$
X_{k}=F_{k} D_{k} A^{t}+E_{k}
$$

subject to $F_{k}^{t} F_{k}=\Phi, k=1, \ldots, K$ where $F_{k}$ is the component matrix in the second mode corresponding to the $k$ th frontal slice, $\Phi$, 
which is the matrix product of $F_{k}$ and its transpose, is required to be invariant for all slices $k=1, \ldots, K$. In equation (3), we observe that unlike in a PARAFAC model, the component matrix in the second mode can vary across slices in a PARAFAC 2 model. This relaxation enables the use of multi-way models in the cases, where a PARAFAC model cannot fully recover the underlying structure. Directly fitting PARAFAC 2 on raw data has more advantages than indirect fitting in terms of imposing constraints, handling missing data and generalization of the model to $\mathrm{N}$-way arrays [5]. This is an objective in this paper too.

\subsection{Fitting PARAFAC2}

A direct method for fitting PARAFAC2 model is proposed by Kiers [5]. In this method by defining new variables problem is defined to minimization of

$$
\sigma_{2}\left(P_{1}, \ldots, P_{k}, F, A, D_{1}, \ldots, D_{k}\right)=\sum_{k=1}^{K}\left\|X_{k}-P_{k} F D_{k} A^{t}\right\|^{2}
$$

over all its arguments. subject to the constraints $P_{k}^{t} P_{k}=I_{R}$, where $I_{R}$ is an $(R \times R)$ identity matrix and $D_{k}$ diagonal for $k=1, \ldots, K$. To minimize this function, an alternative least squares (ALS) algorithm that alternately minimizes equation (4) over $P_{k}$ for fixed $F, D_{k}$ and $A, k=1, \ldots, K$, and minimizes $F, D_{1}, \ldots, D_{K}$ and $A$ for fixed $P_{1}, \ldots, P_{K}$ is used. If the number of rows in the $k$ th data matrix is considerably larger than its columns, it will be inefficient to work with the full data matrix $X_{k}$. In such cases the original indirect PARAFAC2 method, working with crossproduct matrices $X_{k}^{t} X_{k}$, might seem to be more efficient. The direct PARAFAC2 method can be made considerably more efficient in this cases by replacing $X_{k}$ with a smaller matrix with the same cross-product matrix like $H_{k}$. The algorithm for factorization of a $(I \times J \times K)$ three-way data with $R$ common factors may be summarized into the following steps:

Step 0 . If $J<I$, replace $X_{k}$ by $H_{k}$, from the Cholesky decomposition $X_{k}^{t} X_{k}=H_{k}^{t} H_{k}$.

Step 1. Initialize $A$ as the loading matrix from PCA (Principal Component Analysis) on $P_{k} X_{k}^{t} X_{k}$ and initialize $\mathrm{F}$ and $D_{1}, \ldots, D_{K}$ as $I_{R}$.

1a. Compute the SVD $F D_{k} A^{t} X_{k}^{t}=U_{k} \Delta_{k} V_{k}^{t}$ and update $P_{k}$ as $V_{k} U_{k}^{t}, k=1, \ldots, K$.

1b. Update $\mathrm{F}, \mathrm{A}$ and $D_{1}, \ldots, D_{K}$ by one cycle of a normal PARAFAC algorithm applied to the $(R, J, K)$ three-way array with frontal planes $P_{k}^{t} X_{k}, k=1, \ldots, K$.

1c. Evaluate $\sigma_{2}$ from (4). If $\sigma_{2}^{\text {old }}-\sigma_{2}^{\text {new }}>\epsilon \sigma_{2}^{\text {old }}$ for some small value $\epsilon$, repeat Step 1, else go to Step 2.

Step 2. If $X_{k}$ has been replaced by $H_{k}$ in Step 0, now replace $H_{k}$ by $X_{k}$ and compute $P_{k}$ according to Step 1 a for $k=1, \ldots, K$ and exit.

After convergence, as for PARAFAC2, there will be four matrix outputs as follow:

1- $A(I \times R)$ related to the first dimension of $X$.

2- $K P(J \times R)$ related to second dimension of $X$.

3- $C(K \times R)$ related to third dimension of $X$ with each row equal to diagonal elements of $D_{k}$.

4- $F(R \times R)$ as constant matrix.
Unlike PARAFAC which has only one $P$ related to its second dimension, there are $K P$. By this variation the residual error of PARAFAC2 decomposition is lower than the PARAFAC and better signal or factor reconstruction is achieved for signal separation applications.

\section{EEG SEPARATION AND LOCALIZATION}

Separation and localization of brain sources such as brain rhythms have recently been used in BCI applications [7] . On some other researches brain signal separation and localization is along its temporal direction for applications such as eye blink removal and detection and localization of focal epilepsy and epileptic seizure [8]. In these applications linear mixing is used for brain signals:

$$
X=A S+E
$$

where $E \in R_{n \times k}$ is assumed to be a white Gaussian noise vector, $A \in R_{n \times m}$ is the mixing matrix and in our application it includes spatial information of the sources, $S \in R_{m \times k}$ includes $m$ source signals and $X \in R_{n \times k}$ is assumed the matrix of $n$ electrode measurements. Because of using many electrodes for EEG recording $(m<n)$ the system is considered overdetermined. Often the source separation problem changes to an inverse problem formulated as :

$$
Y=W X
$$

where $W \in R m \times n, y \in R m$ and $Y$ are the estimated sources. In most of the separation and localization of brain signals, the localization process starts after completing the separation process.

$$
\hat{A}=X(Y)^{\dagger}
$$

where $\hat{A}$ includes spatial information of the sources and $(.)^{\dagger}$ denotes pseudo inverse operation. During 2-D or 3-D source localization process $\hat{A}$ is used to estimate the spatial information of each source.

\subsection{Using PARAFAC2 for blind source separation and localiza- tion}

Regarding PARAFAC2 decomposition and its outputs for each 3-D data $X(I \times J \times K)$ with $R$ factors there are $A \in R_{I \times R}, K$ number of $F \in R_{J \times R}$ and $C \in R_{K \times R}$ as outputs. If this 3-D $X$ data is the output of temporal segmentation for a 2-D mixed data then $I$ will be the number of electrodes, $J$ will be the number of samples in each temporal segments and $K$ will be the number of segments. The outputs of PARAFAC2 is $A(I \times R)$ which includes mixing matrix or spatial information for $R$ sources and the other outputs estimate the source signals. It is obvious that by using PARAFAC2 decomposition, both sources and their spatial information are achieved simultaneously. It means that PARAFAC2 can be used for blind source separation just by applying temporal segmentation on each 2-D mixture signals. Temporal segmentation can be performed by overlapped or non-overlapped windowing of the signals. However, we show that PARAFAC2 does not need overlapped windows and it can do separation on just reshaped 3-D signal.

Regarding the PARAFAC2 direct fitting method all outputs are computed and updated in each time slice which in our case considered as segment. Dividing the 2-D signal to a number of segments (for example, to more than 10 segments) helps PARAFAC2 to do 


\begin{tabular}{|l|l|l|l|}
\hline SNR & 10 & 14 & 20 \\
\hline Average Error (percent) & 6.93 & 3.44 & 2.68 \\
\hline
\end{tabular}

Table 1. Average spatial error in different SNRs.

signal separation in different slices with a common spatial information. As we know, spatial information of the sources are fixed for all segments of the signals as $A(I \times R)$ and PARAFAC2 tries to decompose each 2-D segment signal with a constant spatial term $A$. The fixed spatial term in all segments and also considered constraint at equation (4) help PARAFAC2 to have a unique solution for overall source separation and localization tasks simultaneously.

\section{EXPERIMENTAL RESULTS}

To show the effectiveness of PARAFAC2 the proposed source localization and separation method is applied to both simulated and real data. We used N-way toolbox for MATLAB [9] in these experiments. What follows is the description of the simulated and real data and the results of applying PARAFAC2 to both datasets.

\subsection{Simulated data results}

Four brain sources are located on a 3-D spherical simulated head model and then by applying the method on their linear mixtures we can localize and separate the sources.

\subsubsection{Forward brain model}

In order to simulate the mixture signals we used a three layer spherical head model. At this model conductivity ratio used for forward solution computation was chosen 1:0.0125:1 for scalp:skull:brain layers. By this model there are 6510 grid points with inter-grid distance of $2 \mathrm{~mm}$ and We placed 25 electrodes on the scalp using standard $10-20$ system[10]. At this model each source can be assigned to one of the grid points with specified 3-D spatial information $(x, y, z)$.

\subsubsection{Source separation}

In this simulation we generated a Theta rhythm signal $(4-8 \mathrm{~Hz})$, an Alpha rhythm signal (7-12Hz), an Eye-blink signal (a Sinc function signal in front of head) and an ERP (Evoked related potential) signal. We used $100 \mathrm{~Hz}$ sampling frequency and also we put these signals at different 3-D locations within forward brain model. We did the separation in two experiments with additive Gaussian noise to mixture signals, first at $S N R=20 \mathrm{~dB}$ and second at $S N R=10 \mathrm{~dB}$. Figure 2(a) shows the original signals and their mixing matrices on 2-D map as spatial information on scalp topography plots. the numbers shown on the scalp are the electrode numbers and it can be seen that some sources are localized between the electrodes, e.g. the second source is located between electrodes 3 and 22. Figure 1 shows the original and the estimated mixing gains or spatial information for second source in the above two cases. It can be seen that our localization error for first case is close to zero but this error rate varies for different sources. Table 1 compares the average spatial error for different SNRs. In the case of estimated source signals Figure 2(b) shows the estimated sources and at their 2-D spatial information for second noisy case.

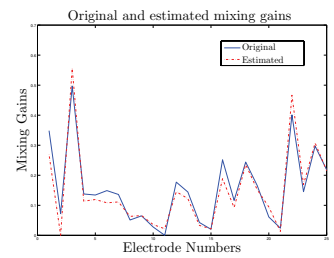

(a)

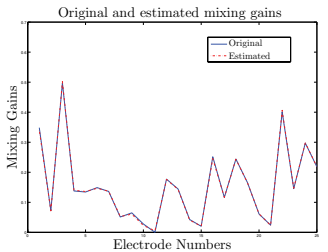

(b)
Fig. 1. (a). Original and estimated mixing gains for the second source ( $\mathrm{SNR}=10 \mathrm{~dB})$. (b). Original and estimated mixing gains for the second source $(\mathrm{SNR}=20 \mathrm{~dB})$.
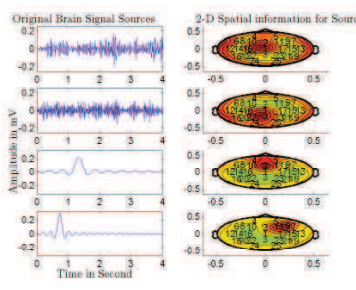

(a)

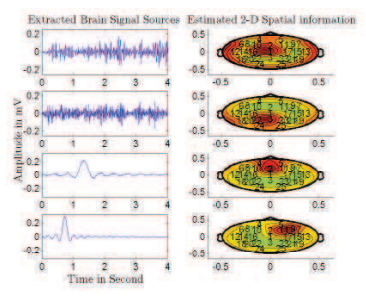

(b)
Fig. 2. (a). Original signals and their spatial information. (b) Separated sources and their estimated spatial information for $\mathrm{SNR}=10 \mathrm{~dB}$.

According to our previous discussion we need to make a 3-D signal from our 2-D signal by temporal segmentation. Here we perform the separation for 100 seconds of 25 mixture signals (channels) and we selected 1 second length for each segment. The result of segmentation or reshaping the 2-D signal $\mathrm{X}(25,10000)$ is a 3-D tensor $\mathrm{X} 3(25,100,100)$ The estimated sources and their spatial information are shown in Figure 2(b). Also, according to the synthetic signals generated for two different brain rhythms, there is frequency overlap between $4-10 \mathrm{~Hz}$ and $8-12 \mathrm{~Hz}$ signals which does not affect the quality of separation process because here only spatial disjointedness and linear independency between the sources are necessary to have an effective separation.

\subsection{Real data results}

In order to evaluate the PARAFAC2 source separation on real EEG signals We used dataset-1 of BCI Competition IV database [11]. Signals from 59 EEG positions were measured and bandpass filtered between 0.05 and $49 \mathrm{~Hz}$ during normal motor imagery tasks. We picked 30 seconds of this signals which included few eye-blinks. The signals for the first 3 electrodes are shown in Figure 3(a).

We do not have any exact information about the number of active sources. However we clearly see one powerful eye-blink source and it is expected that after separation it will come up as one of the dominant signals. We used PARAFAC2 blind source separation for finding different number of sources and it resulted that for a number of sources greater than 8 the eye-blink signal comes up as one of the three first powerful sources and for lower number of sources the result sometimes shows an eye-blink signal and another powerful signal. The results for 6 sources are shown in Figure 3(b)(c).

The estimated location of the eye-blink is exactly in front of the 


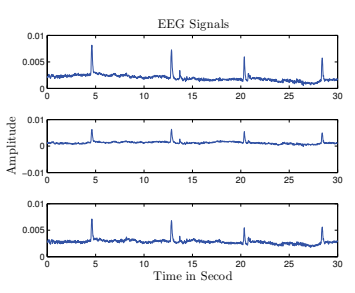

(a)

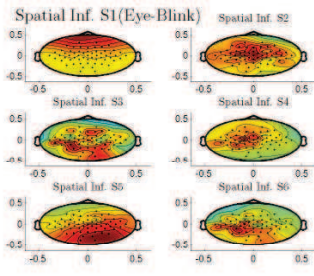

(b)

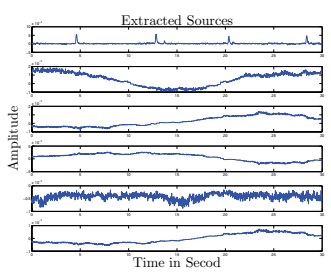

(c)
Fig. 3. (a). First 3 electrodes of 59 EEG electrodes signal including strong eye-blink. (b). Estimated spatial information for Eye-blink and other separated sources. (c). Eye-blink and other separated sources.

head and it can be seen at Figure 3(b). In the case of separation with greater number of sources (e.g. 9,10) the eye-blink signal appears as one of strongest sources again. In another experiment for real data signals we used PARAFAC2 to separate maternal and fetal ECG source signals. The signals from well-known DaISy fetal ECG database is used for our experiment [12].

The database consists of five abdominal and three thoracic channels recorded from the abdomen and chest of a pregnant woman with a sampling rate of $250 \mathrm{~Hz}$. As we need only linear mixtures we selected 3 abdominal electrode signals (electrodes 2,3,5) for our separation. We used 0.1 second time duration segments for temporal segmentation process. Three channels of the dataset may be seen in Figure 4(a). In Figure 4(b) the separated signals are shown. It can be seen that in the estimated maternal signal there is no noticeable effect of fetal ECG and vice versa.

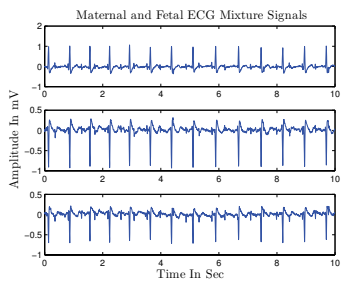

(a)
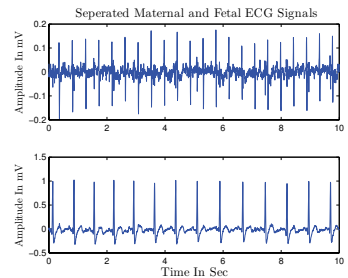

(b)
Fig. 4. (a). Three electrodes of eight electrode mixed ECG signals. (b). Separated maternal and fetal ECG signals.

\section{DISCUSSION AND CONCLUSIONS}

In this paper a PARAFAC2 based method is proposed to separate and localize the brain sources. In this method the mixing matrix and the sources are estimated in each iteration. The mixing matrix information can be mapped to show the locations of the sources in the topography plots over the scalp. It is shown that this method simply does separation only on reshaped version of zero-lagged two dimensional mixture signals and there is no need to have any computation on different lags. As for the temporal segmentation process, there is no need to choose overlapped segments. These features make PARAFAC2 a simple and effective method for blind source separation. Moreover, it is discussed that this method extracts the spatial information of the sources during the source estimation. Physically PARAFAC2 relies on disjointedness of the sources in some domains rather than other properties such as independency and uncorrelatedness. Also, in presence of forward model of head we can obtain the 3-D locations of the sources by an inverse problem solving on estimated spatial informations. We also evaluated this method for maternal and fetal ECG signal separation.

\section{REFERENCES}

[1] S. Sanei and J. Chambers, EEG signal Processing, John Wiley and Sons, 2007.

[2] N. Ryan, M. Mesbah, and B. Boashash, "Seizure detection of newborn EEG using a model based approach: a review of performance," Signal Processing and Its Applications, 1999. ISSPA '99. Proceedings of the Fifth International Symposium on, vol. 1, pp. 243-246, 1999.

[3] J. Z. Liu, B. Yao, B. E. Lewandowski, C. Karakasis, R. W. Brown, and G. H. Yue, "Source Localization of EEG Signals during Muscle Fatigue," APS Meeting Abstracts, Oct. 2003.

[4] R. Bro, "PARAFAC. tutorial and applications," Chemometrics and Intelligent Laboratory Systems, vol. 38, no. 2, pp. 149 171, 1997.

[5] H. A. L. Kiers, J. M. F. Ten Berge, and R. Bro, "PARAFAC2 - Part I. A direct fitting algorithm for the PARAFAC2 model," Journal of Chemometrics, vol. 13, pp. 275-294, 1999.

[6] R. A. Harshman, "PARAFAC2: Mathematical and technical notes," UCLA Working Papers in Phonetics, vol. 22, pp. 30-44, $1972 b$.

[7] K. Nazarpour, P. Praamstra, R. C. Miall, and S. Sanei, "Steadystate movement related potentials for brain computer interfacing," Engineering in Medicine and Biology Society, pp. 53105313, Aug. 2008.

[8] J. Corsini, L. Shoker, S. Sanei, and G. Alarcon, "Epileptic seizure predictability from scalp EEG incorporating constrained blind source separation," Biomedical Engineering, IEEE Transactions on, vol. 53, no. 5, pp. 790-799, May 2006.

[9] R. Bro and A. Claus, "The N-way toolbox for MATLAB," http://www.models.life.ku.dk/source/nwaytoolbox/index.asp.

[10] H. Mohseni, E. Wilding, and S. Sanei, "Sequential monte carlo techniques for EEG dipole placing and tracking," Sensor Array and Multichannel Signal Processing Workshop, pp. 95-98, July 2008.

[11] "BCI competition iv, data set 1 , http://ida.first.fhg.de/projects/bci/competition-iv," .

[12] "Daisy fetal ECG database, http://www.dcsc.tudelft.nl/ bdeschutter/pub/rep/97-70.pdf," . 